International Journal of Wireless \& Mobile Networks (IJWMN) Vol. 3, No. 5, October 2011

\title{
Design and Performance Evaluation of OFDM- Based Wireless Services Employing Radio over Optical Wireless Link
}

\author{
Jiang Liu, Wasinee Noonpakdee, Shigeru Shimamoto \\ Global Information and Telecommunication Institute, Waseda University, \\ 1-3-10, Nishi-Waseda, Shinjuku-Ku, Tokyo, 169-0051 Japan \\ liujiang@aoni.waseda.jp, wasinee@toki.waseda.jp, shimalwaseda.jp
}

\begin{abstract}
This paper studies an Orthogonal Frequency Division Multiplexing (OFDM) based wireless services employing Radio over Optical Wireless (RoOW) to get a high performance transmission while eliminating the drawback of possible radio wave interference to electro-medical apparatus in indoor communication system. An optical Intensity-modulated Direct-detection (IM/DD) system using RF subcarrier modulation is considered and OFDM RF signals are assumed as the subcarrier. The transmission performance is evaluated by simulation considering the influence of peak clip of OFDM signal and different radio fading scenarios. Simulation result shows that the modulation index $m$, optical transmit power, radio environments are important factors which affect the communication performance significantly. It also shows when the received optical power is larger than-20dBm, which is a very practical power level, the value of BER becomes almost independent of the optical power and the radio environment becomes more important. That means the indoor optical wireless communication (OWC) has high transmission performance and the proposed relay system is a very available system as long as the radio environment is eligible. We believe that the proposed system is a practical system and can provide a low-cost, high-quality wireless services for $R F$ sensitive areas.
\end{abstract}

\section{Keywords}

Optical Wireless Communication, Relay Station, Intensity-modulated Direct-detection (IM/DD), RF Subcarrier, Diversity

\section{INTRODUCTION}

Mobile terminals including wireless voice telephone, mobile internet access, video calls and mobile TV have become more and more necessary in our daily life. Right from a school going child, a house wife to a servant, the mobile terminals has its major impact on their lives [1]. Users want to use them without any spatial constraint because they have become indispensable tools. However, the electromagnetic interference with electro-medical apparatus generated by using radio frequency (RF) communication becomes a health issue with the increasing number of mobile users. In medical facilities, such as hospitals, clinics, and doctor's offices, sensitive equipments may malfunction if exposed to high RF powers, possibly endangering patients' lives [2]-[4]. Therefore, in medical institutions, mobile phones are 
required to be turned off except at some special designated places. Similarly, all railroad companies demand that mobile phones should be turned off nearby priority seats. However there are so many mobile phone users that are difficult to be convinced to turn off their mobile phones.

Optical wireless communication (OWC) system is safe to electro-medical apparatus and can keep the convenience of current wireless communications using radio waves. Moreover, OWC has features like unlimited license-free bandwidth, cheaper transceivers, high security etc [5][10], which is suitable to RF sensitive service areas. As a feasible application of OWC, we propose a relay scheme for an indoor optical wireless communication system tends to provide non-RF communication for the last few meters to/from mobile terminals operating in an RFsensitive area. It also can provides applications for the dead zones where wireless signal from base station cannot reach or is very weak such as tunnel, areas behind buildings or mountainous places etc.

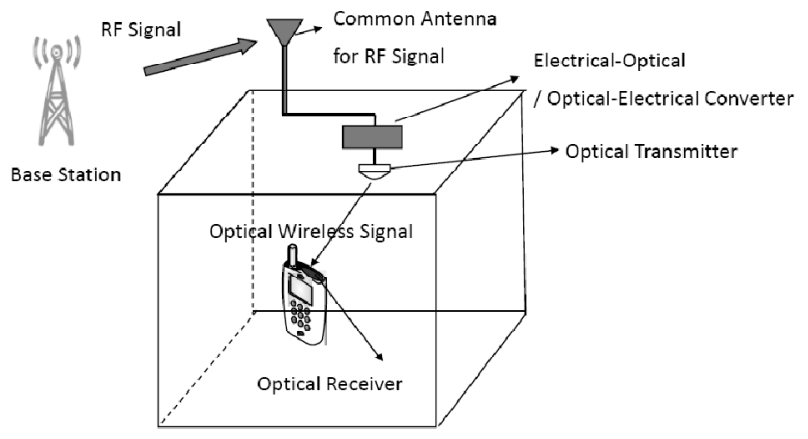

Fig. 1 Relay System Model

Orthogonal frequency division multiplexing (OFDM) is one of the most popular techniques for high data rate wireless communication and is great interested by the researchers in the universities and research laboratories all over the world. It is an effective high speed data transmission scheme without using very expensive equalizers and it has been proposed as the air interface for broadband wireless applications [11]. OFDM has been accepted in the IEEE802.11a local area network (LAN), IEEE802.16a local area network /Metropolitan area network (LAN/MAN), High Performance Local Area Network type 2 (HIPERLAN/2), standards, and Mobile Multimedia Access Communication (MMAC) Systems. OFDM is also being considered in IEEE802.20a, a standard in the making for maintaining high-bandwidth connections to users moving at speeds up to $60 \mathrm{mph}[12]$, [13]. OFDM is considered a potential candidate for the next-generation mobile wireless systems [14], [15].

This paper presents an OFDM-Based Wireless Services for indoor RF sensitive area. One of the main disadvantages of OFDM is its high peak-to-average power ratio (PAPR) [11] and high amplitude peaks may be clipped. This system is quantitatively evaluated considering the peak clip. Moreover, the BER performance is studied in different fading scenarios.

The remainder of this paper is organized as follows. System model is introduced in section 1 . The related work is given in section 2 and the system model is discussed in section 3. In Section 4, we present the mathematical modeling for the transmission of OFDM signals over RoOW link. The analytical results are shown and discussed in Section 5. Finally, Section 6 concludes the paper. 
International Journal of Wireless \& Mobile Networks (IJWMN) Vol. 3, No. 5, October 2011

\section{RELATED WORK}

There are two methods which can be considered to relay RF signals to mobile terminal inside the service area. One is demodulating the information data to baseband signal and use optical digital modulation schemes such as on-off-keying (OOK) modulation. However, each mobile user in a service area may use different RF broadband due to have a contract with different provider. It is difficult to perform the regeneration process at the relay station. Moreover, multiple access schemes have to be considered for multiple user case. Another relay method is using non-regeneration system. If non-regeneration system is used, only a minimal set of frontend hardware components is needed in the receiver/transmitter functions at the relay station. In this paper, a non-regeneration system is adopted. We propose an optical intensity-modulated direct-detection (IM/DD) [16] relay scheme for an indoor optical wireless communication system, referred to as Radio over Optical Wireless (RoOW).

Radio transmission over optical fiber links, referred to as radio-over-fiber (RoF), has been viewed as a way of simplifying the architecture of remote antenna base-stations and realizing high-performance networks [17]. Similar to RoF, Transmission of RF using OWC link provides as a way of simplifying the architecture of relay station. It combines the advantages of high transmission capacity enabled by optical device technologies and easy deployment of wireless links. By using the proposed RoOW scheme, at the relay station, the received RF signals are directly modulated with IM, up-converted into optical band, and propagated as optical signals. The RF signal is defined to be RF subcarrier in this paper. The details of the proposed system model will be introduced in next section.

\section{SYSTEM MODEL}

In this paper, we propose a relay system using OWC as shown in Figure 1. This model proposes to set up a relay station for mobile phone coverage inside medical institutions, trains, apartments, etc. It is proposed to keep the present wireless access from base station, and replace the radio signal link between the relay station and the mobile terminal with optical wireless signals. The conventional RF wireless communication is used between the relay station and the base station. For the relay station, the RF transceiver is set up outside the service area, and the optical transceiver is set up on the ceiling or the wall inside the service area.

We propose to use OWC both for downlink and uplink. The wavelength of the optical wireless signal used in the downlink is different from the wavelength of optical wireless signal used in the uplink. The downlink from the base station to the mobile terminal is established in two steps (refer to Figure 1). Firstly the relay station receives RF signals; secondly the received $\mathrm{RF}$ signals are converted into optical signals and sent to the mobile terminal using light emission elements. In the uplink, mobile terminal convert RF signal to optical signals and send it to optical receivers inside the room. The RF signals are extracted from the received optical wireless signals in the relay station, and then sent to the base station as uplink signals. The RF signals sent from the relay station can be sent at a higher power level compared with the RF signals sent directly from the mobile terminal to achieve higher transmission quality. Moreover, in the downlink, better than RF relay method, if mobile terminal can also receive some RF signals from the base station, there are no interference between the RF signals and the optical wireless signals due to different transmission band. 
International Journal of Wireless \& Mobile Networks (IJWMN) Vol. 3, No. 5, October 2011

Since RF signals are not converted to base band signals in the proposed non-regenerative relay station, the circuits added to the relay station can be reduced. Therefore, we believe that the proposed system using such a combination can save electromagnetic interference with electro-medical equipment in the coverage area without changing the present communication infrastructure.

\section{Mathematical modelling for the transmission of OFDM signals over RoOW link}

We consider an optical IM/DD system using RF subcarrier modulation and OFDM signal is assumed as the RF subcarrier signal. The configuration of OFDM signal transmission over optical wireless link is shown as Figure 2. An OFDM carrier signal is the sum of a number of orthogonal sub-carriers, with baseband data on each sub-carrier being independently modulated commonly using some type of quadrature amplitude modulation (QAM) or phase-shift keying (PSK). In this paper, QPAK modulation is assumed. A serial data stream of binary digits is first multiplexed into parallel streams, and each one mapped to a symbol stream using QPSK modulation constellation. IFFT and FFT are implemented at the transmitter and the receiver respectively.

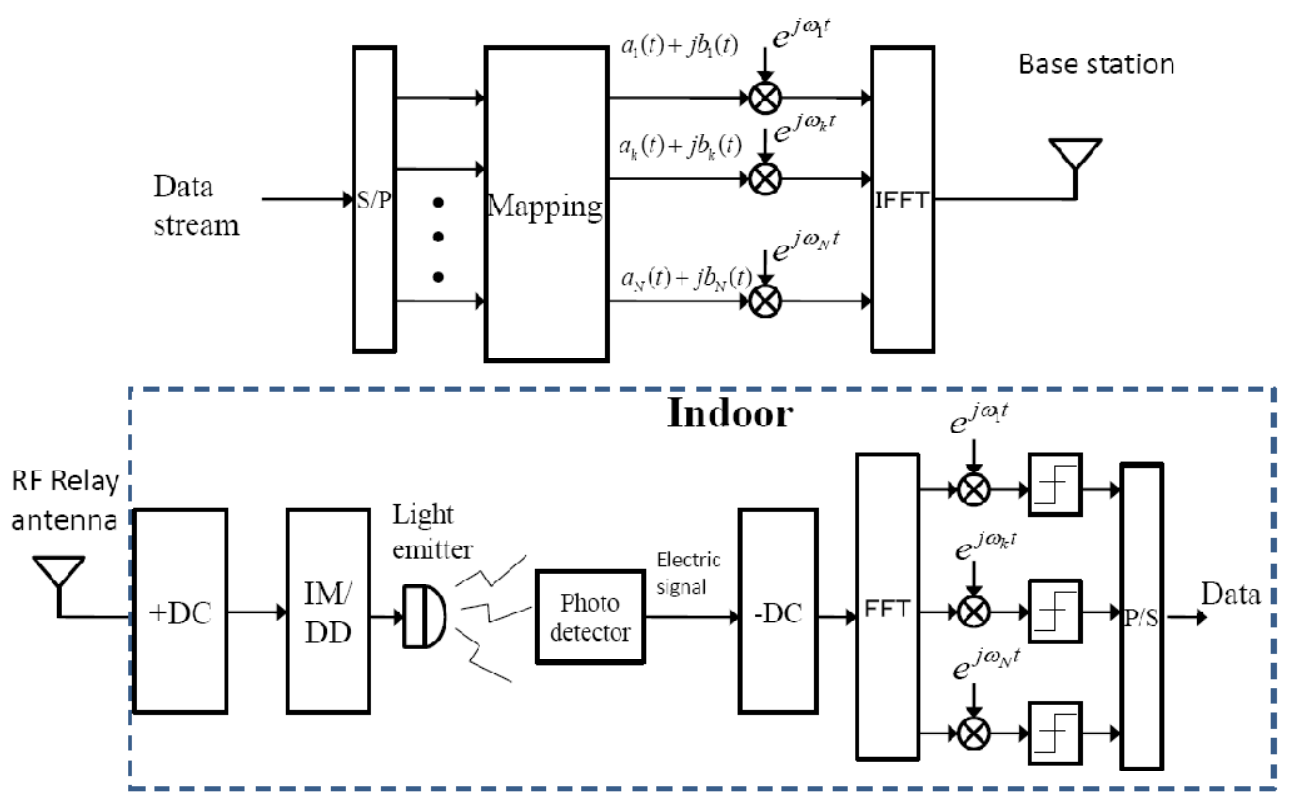

Fig. 2 Configuration of OFDM signal transmission over optical wireless link.

\subsection{IM/DD optical channel}

In the OFDM transmission system, the data stream is divided into $N$ narrowband data streams, $N$ corresponding to the subcarriers. That is to say one OFDM symbol consists of $N$ symbols. The amplitude of the OFDM baseband signal at each sample point $t=n \Delta T$ in the effective symbol period is shown as: 
International Journal of Wireless \& Mobile Networks (IJWMN) Vol. 3, No. 5, October 2011

$$
x(n \Delta T)=\sum_{k=0}^{N-1}\left\{d_{k} e^{j 2 \pi \frac{n k}{N}}\right\}=\sum_{k=0}^{N-1}\left\{a_{k} \cos \left(2 \pi \frac{n k}{N}\right)+j b_{k} \sin \left(2 \pi \frac{n k}{N}\right)\right\}
$$

where $n=t / \Delta T, a_{k}+j b_{k}$ is a complex symbol of $k$ th subcarrier after mapping. The OFDM signal for $N$ subcarriers, after up-conversion to the wireless service carrier frequency $f_{R F}$, can be written as:

$$
S_{R F}(t)=\sum_{k=0}^{N-1}\left\{a_{k} \cos \left(2 \pi \frac{n k}{N}\right) \cos \left(2 \pi f_{R F} t\right)+j b_{k} \sin \left(2 \pi \frac{n k}{N}\right) \sin \left(2 \pi f_{R F} t\right)\right\}
$$

In this paper, the signal is assumed to be intensity modulated in a linear area of the light emission element. The average absolute value of the amplitude of $a_{k}+j b_{k}$ is assumed to be one. The optical transmission signal after fixed direct current (DC) component is added can be expressed as [18]:

$$
P_{t}(t)=P_{o p t}\left\{1+m \cdot\left(S_{R F}(t)+n_{R F}\right)\right\}
$$

Here, $P_{\text {opt }}$ represents the average power radiated by the light emission element. $n_{R F}$ is an additive white Gaussian noise (AWGN) to the RF signal, and $m$ is the modulation index. When $m=0$, no modulation of the carrier is performed. The absolute value of $m S_{R F}$ is smaller than 1 because the optical signal is nonnegative. If $m S_{R F}$ is greater than 1, the carrier is actually cut off for some period of time, and unwanted harmonics are created at the transmitter output.

In this paper, Lambetian radiation intensity pattern is considered. Supposing the considered indoor OWC is performed in a relatively large space, reflection light is neglected due to its lower energy comparing to line of sight (LOS) light at the receiver side. The intensity optical channel of this system is:

$$
h=\frac{1}{2 \pi} \cos \theta \cos \psi \operatorname{rect}(\theta / F O V) \frac{A_{O R}}{R_{d}^{2}} \delta\left(t-R_{d} / c_{o}\right)
$$

$\theta$ is the angle between the beam central direction and the line between the transmitter and receiver. $\psi$ is the angle between the direction vertical to the aspect of the optical detector and the line between the optical transmitter and receiver. $R_{d}$ is the distance between the optical transmitter and receiver, $A_{O R}$ is the photosensitive area of the optical detector where the incident radiation is collected and converted to an electronic signal. FOV is the angle of field of view. $c_{o}$ is light speed. $\delta\left(t-R_{d} / c_{o}\right)$ is the delayed function due to the signal propagation. $\operatorname{rect}(x)$ is the rectangular function defined as:

$$
\operatorname{rect}(x)=\left\{\begin{array}{l}
1 \text { for }|x| \leq 1 \\
0 \text { for }|x|>1
\end{array}\right.
$$

Then the received optical power $P_{R}$ at the mobile terminal is given by: 
International Journal of Wireless \& Mobile Networks (IJWMN) Vol. 3, No. 5, October 2011

$$
P_{R}=P_{t} \otimes h=P_{e}\left\{1+m \cdot\left(S_{R F}+n_{R F}\right)\right\}
$$

Here, $P_{e}$ is the average received optical power. $n_{R F}$ is the Additive White Gaussian Noise (AWGN) at the RF transceiver of relay system, and $m$ is the modulation index.

Assuming that the DC term $P_{e}$ can be eliminated at the output of the optical receiver, the received information signal $S_{\text {sig }}$ coming from the RF signal is:

$$
S_{s i g}=S_{R F} P_{e} m r
$$

Here, $r$ represents the photodetector responsively. The average power of the information signal $P_{\text {sig }}$ is

$$
P_{\text {sig }}=P_{s}\left(P_{e} m r\right)^{2}
$$

where $P_{s}$ is the average power of signal $S_{R F}$.

\subsection{Noise model and signal to noise ratio (SNR) analysis}

In this paper, we assume that the noise affecting the optical parts of our system is generated at the optical receiver. Here, Avalanche Photo Diode (APD) is assumed to be the optical receiver. Shot noise $\sigma_{\text {shot }}^{2}$ and thermal noise $\sigma_{t h}^{2}$ are considered in our analysis. Both shot noise and thermal noise are modelled as additive white Gaussian noise (AWGN) with a double-sided power spectral density $\sigma^{2}$. The expression of the noise variance is given by

$$
\begin{aligned}
\sigma^{2}{ }_{\text {opt }} & =\sigma_{\text {shot }}^{2}+\sigma_{\text {th }}^{2} \\
\sigma^{2}{ }_{\text {shot }} & =2 q r P_{e} B+2 q r p_{b g} \Delta \lambda_{n b} A_{b g} B \\
\sigma_{t h}^{2} & =\frac{4 k T B}{R_{L}}
\end{aligned}
$$

Here, (10) includes the shot noise from the background light and the shot noise from the optical signal. Each parameter in (10) (11) are described in table 1.

For the noise of the whole process, it should include the noise generates when the RF signal is received and when the optical signal is received. The SNR from the base station to the mobile terminal can be shown as:

$$
\Gamma_{\text {all }}=\frac{P_{s}\left(P_{e} m r\right)^{2}}{\left(P_{e} m r\right)^{2} \sigma_{R F}^{2}+\sigma_{o p t}^{2}}
$$

$\sigma_{R F}^{2}$ is the noise generated at the relay station when radio signal is received.

If the SNR of the transceiver of the relay station is defined as $\Gamma_{a n t}, P_{s}$ is given as 
International Journal of Wireless \& Mobile Networks (IJWMN) Vol. 3, No. 5, October 2011

$$
P_{s}=\sigma_{R F}^{2} \Gamma_{a n t}
$$

Form (12)-(13), $\Gamma_{\text {all }}$ can be rewritten as:

$$
\Gamma_{\text {all }}=\frac{\left(P_{e} m r\right)^{2} \sigma^{2}{ }_{R F}}{\left(P_{e} m r\right)^{2} \sigma_{R F}^{2}+\sigma^{2}{ }_{o p t}} \Gamma_{a n t}
$$

Therefore, the ratio $K_{S N R}$ of $\Gamma_{\text {ant }}$ to $\Gamma_{\text {all }}$ can be expressed as follows:

$$
K_{S N R}=\frac{\left(P_{e} m r\right)^{2} \sigma_{R F}^{2}}{\left(P_{e} m r\right)^{2} \sigma_{R F}^{2}+\sigma_{o p t}^{2}}
$$

Table1. Parameters of shot noise and thermal noise

\begin{tabular}{|c|c|c|}
\hline Symbol & Quantity & Value \\
\hline$A_{b g}$ & Detector area & $1(\mathrm{~cm} 2)$ \\
\hline$\Delta \lambda_{n b}$ & Noise-bandwidth factor & $30(\mathrm{~nm})$ \\
\hline$q$ & Electron charge & $1.6 \times 10-19(\mathrm{C})$ \\
\hline$r$ & Photodetector responsively & $0.53(\mathrm{~A} / \mathrm{W})$ \\
\hline$p_{b g}$ & Background irradiance & 5.8 \\
\hline$k$ & Boltzmann's constant & $1.374 \times 10-23(\mathrm{~J} / \mathrm{K})$ \\
\hline$T$ & Temperature & $300(\mathrm{~K})$ \\
\hline$R_{L}$ & Load resistance & $240(\Omega)$ \\
\hline$B$ & Filter bandwidth & $1 \mathrm{M}(\mathrm{Hz})$ \\
\hline
\end{tabular}

\subsection{Channel model in fading environment}

It is necessary to consider the propagation environment of the RF signal and the indoor optical wireless signal because our system is composed of two parts that use an existing RF communication and an optical wireless communication. In our paper, assuming people indoor are moving slowly and the power of multipath light is very low, we ignore light fading. In outdoor radio communications, because the antenna for the RF wave of the relay station is set up outside, we may assume that we are having a Rayleigh fading channel or Rice fading channel. $K_{f}$ is defined as the Rice factor [19]. In indoor radio communications, normally we consider that we are having a Rayleigh fading channel. 


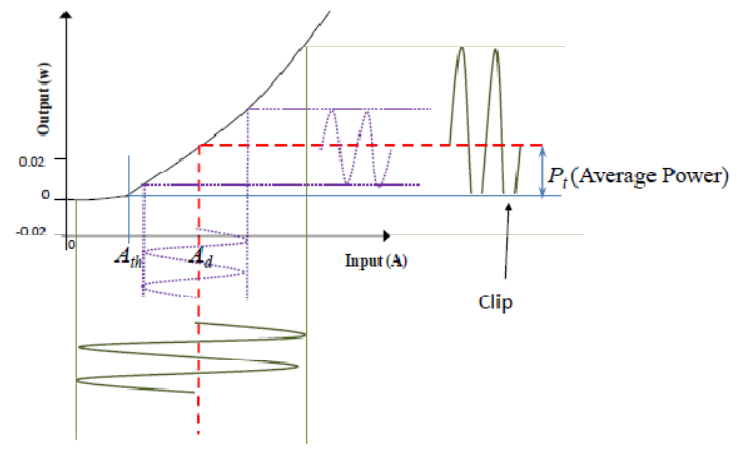

Fig. 3 Output Character of LD

\section{Evaluation of characteristics of proposal method by simulation}

According to the study give in the previous section, in order to know the feasibility of the proposed system and to analyze the bit error rate (BER), the RF noise ratio compared to the entire system noise, the power of the optical signal, the SNR of the RF transceiver and the fading environment, the peak clip of OFDM signal must be considered. In this chapter, the BER performance considering peak clip of OFDM signal and different radio wave environments is evaluated by simulation.

PAPR is one of the main drawbacks of OFDM system. Shown as Figure 3, when PAPR is high, peak clip will happen and influence the transmission characteristic because RF signal is intensity modulated to optical wireless signal. In this paper, we assume that the positive peak of the RF signal is completely modulated to optical signal, and the negative peak will be clipped when the threshold is exceeded. If we define the threshold current value of LD (Laser Diode) output, the bias current added to the signal, the modulation index of the intensity modulation (refer to function (3)), the amplitude of the OFDM signal as $A_{t h}, A_{d}, m, A_{s}$, the condition that the peak clip does not happen is shown as:

$$
m A_{s}<1-A_{t h} / A_{d}
$$

Table 2 Parameters of simulation

\begin{tabular}{|c|c|}
\hline Number of FFT points & 128 \\
\hline Number of sub channels: $\mathrm{k}$ & 128 \\
\hline Length of Pilot & 32 \\
\hline Modulation & QPSK \\
\hline Symbol rate & $250000 \mathrm{bps}$ \\
\hline Synchronization & Perfect \\
\hline Channel Estimation & Perfect \\
\hline
\end{tabular}


International Journal of Wireless \& Mobile Networks (IJWMN) Vol. 3, No. 5, October 2011

Figure 4 is the simulation result for $A_{t h} / A_{d}=0.33$ and $P_{e}=-15 \mathrm{dBm}$. The simulation parameter is shown in table 2. From Figure 4, it can be confirmed that the BER characteristic improves as $\Gamma_{\text {ant }}$ grows. Moreover, it is clearly shown that $m$ in Equation (3) infects the transmission performance very much. With the increase of $m$, the BER characteristic can be separated to three regions where the BER performance relatively increases, then keep unchanged, finally deteriorates. First, as $m$ increases, the BER performance improves since $m$ increases the ratio of information signal power to the total optical power. However after $m$ becomes bigger than a certain threshold, the BER performance changes slowly because $m$ cannot change the channel impairments due to the radio noise generated at RF relay station. When $m$ is very large, the pick clip happens and BER performance is decreased.

Figure 5 shows the BER performance versus $P_{e}$ for $\Gamma_{a n t}=10 \mathrm{~dB}$. From this figure, we can see that the higher value of $K_{f}$, the lower values of BER can be obtained. Furthermore, when the average optical receiving power $P_{e}$ is low, the value of BER changes rapidly by change $P_{e}$. When $P_{e}$ is larger than $-20 \mathrm{dBm}$, the value of BER becomes almost independent of $P_{e}$. That is to say, the radio environment of the RF transceiver become more important than $P_{e}$ to BER when $P_{e}$ is larger than $-20 \mathrm{dBm}$.

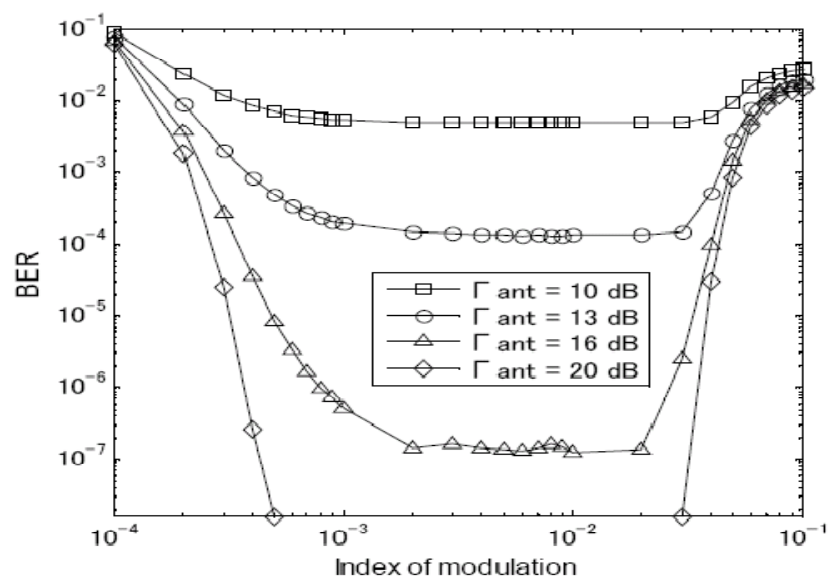

Fig.4 BER via index of modulation $\left(P_{e}=-15 \mathrm{dBm}\right)$

The transceiver of the relay station for RF signals is proposed to be located at a suitable place to make it easier to receive the preferable wave from the base station. The SNR of the RF antenna at the relay station can be much better than the SNR of the conventional RF antenna at our mobile terminal. Here, the SNR of the relay RF antenna is defined as $\Gamma_{a n t}$ and the SNR of the RF antenna of the mobile terminal is defined as $T_{m}$. Figure 6 shows BER performance versus received average optical power $P_{e}$ and SNR for different ratio of $\Gamma_{a n t}$ to $\Gamma_{m}$ in a Rayleigh fading channel when using the proposed relay system and the conventional system. From this figure, it is clear that the same as Figure 5, when $P_{e}$ is bigger than a threshold, the radio environment of the RF transceiver become more important than optical link. That is to say, when the RF environment is available and the received optical power is big enough, the relay system can provide an available communication. It also can be seen that the BER performance can be improved by using higher quality RF relay antenna. 
International Journal of Wireless \& Mobile Networks (IJWMN) Vol. 3, No. 5, October 2011

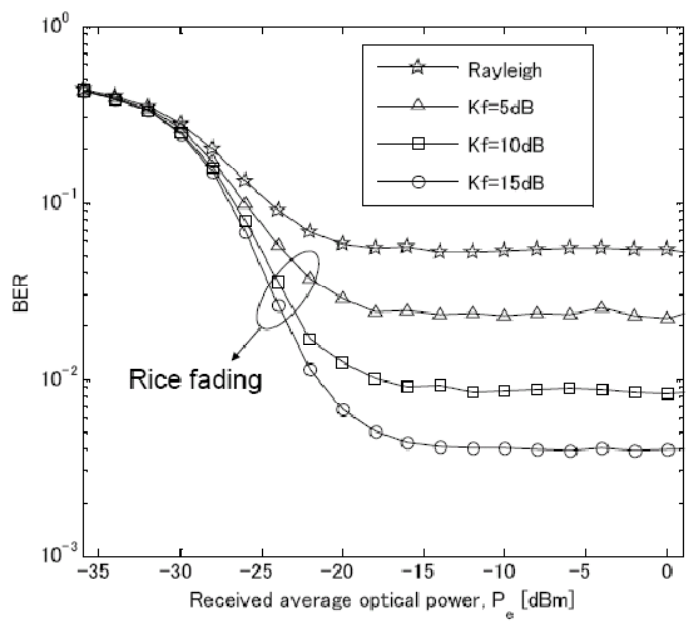

Fig.5 BER via received average optical power $P_{e}\left(\mathrm{~m}=0.001, \Gamma_{a n t}=10 \mathrm{~dB}\right)$

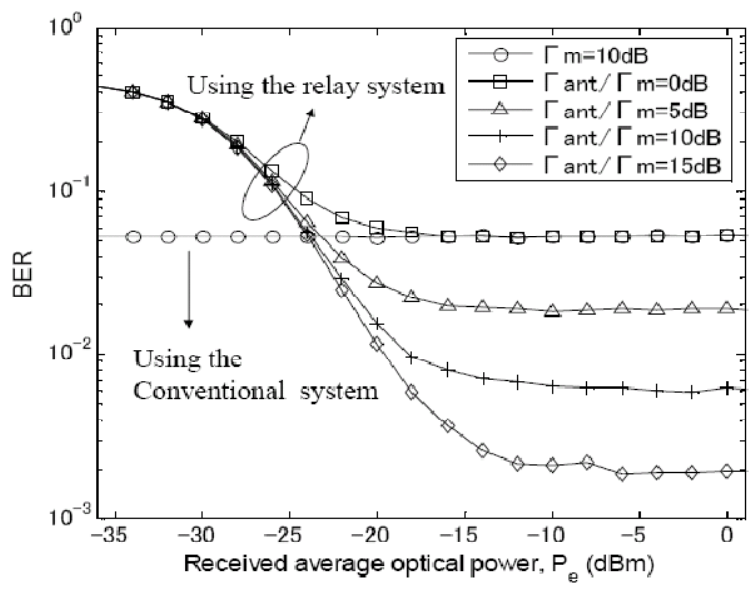

Fig.6 BER via received average optical power $P_{e}$ and $\mathrm{SNR}\left(m=0.001, \Gamma_{m}=10 \mathrm{~dB}\right)$

\section{Conclusion}

In this paper we have proposed a relay system with RF subcarrier based on optical IM/DD channel. First, the advantages of using OWC have been described. Second, the concept of RoOW has been proposed. Then BER was calculated considering the possible peak clip of OFDM signal and different wave environments. Simulation result showed that the modulation index $m$ significantly affects the peak clip of OFDM signal. It also showed that optical transmit power, radio environments such as Rayleigh fading, Rice fading are important factors which affect the transmission performance of the proposed system. It should be noted that when the received optical power is larger than $-20 \mathrm{dBm}$, which is a very practical power level, the value of BER becomes almost independent of the optical power and the radio environment of the RF signal become more important. That means the indoor OWC has very high transmission quality and proposed relay system is a very available system as long as the radio environment is eligible. We believe it can provide practical wireless services for RF sensitive areas. 
International Journal of Wireless \& Mobile Networks (IJWMN) Vol. 3, No. 5, October 2011

\section{ACKNOWLEDGEMENTS}

The authors wish to thank the anonymous reviewers for their valuable suggestions.

\section{REFERENCES}

[1] DR. Tsrmurthy and D. Siva Rama Krishna, "Analysis of cell phone usage using correlation techniques", International Journal of Wireless \& Mobile Networks (IJWMN) Vol. 3, No. 2, April 2011

[2] Eisuke hanada, Tohru Kubota, Hiroaki Shimokawa, "Permission of cellular-phone use in university hospital" $17^{\text {th }}$ Autumn conference of Japanese Society of Medical Electronics and Biological Engineering, 0S15-01, Oct.2003

[3] Yoshiaki Tarusawa, Kohjiroh Ohshita, Yasunori Suzuki, Toshio Nojima, and Takeshi Toyoshima, "Experimental Estimation of EMI From Cellular Base-Station Antennas on Implantable Cardiac Pacemakers" IEEE Transactions on Electromagnetic Compatibility, VOL. 47, NO. 4, NOVEMBER 2005.

[4] Hiroshi Takano, Yasunori Futatsugi, Shigeru Shimamoto, Kiyotaka Seki, "Studies on Optical Wireless Communications in Train and Optical Signal Propagation Model" Transmission,'IEICE Trans. Communications, Vol.J87-B， No.7， pp.950-962，July.2004

[5] Carruthers, J.B. and J.M. Kahn, Angle diversity for nondirected wireless infrared communication. IEEE Transactions on Communications, 2000. 48(6): p. 960-969.

[6] O'Brien, D.C., et al. High speed integrated optical wireless transceivers for in-building optical LANs. in Optical Wireless Communications III. 2000. Boston: SPIE.

[7] Jivkova, S. and M. Kavehrad, Holographic optical receiver front end for wireless infrared indoor communications. Applied Optics, 2001. 40(17): p. 2828-35.

[8] J.R. Barry, Wireless infrared communications, Kluwer, Boston,1994.

[9] H. Willebrand and B. Ghuman, Free Space Optics: Enabling Optical Connectivity in Today's Networks. London, U.K.:Sams, 2002.

[10] V. W. S. Chan, "Free-space optical communications", J. Lightwave Technol., vol. 24, no. 12, pp. 4750-4762, Dec. 2006.

[11] Pawan Sharma and and Seema Verma, "PAPR reduction of OFDM signals Using selective mapping with TURBO code", International Journal of Wireless \& Mobile Networks (IJWMN) Vol. 3, No. 4, August 2011

[12] R. V. Nee and R. Prasad, OFDM for Wireless Multimedia Communications. Norwell, MA: Artech House, 2000.

[13] Jayakumari.J, "MIMO-OFDM for 4G Wireless Systems", International Journal of Engineering Science and Technology, Vol. 2(7), pp. 2886-2889, 2010

[14] Xiaodong Wang, "OFDM and its application to 4G", 14th Annual WOCC 2005. International Conference on Wireless and Optical Communications, 2005. 
International Journal of Wireless \& Mobile Networks (IJWMN) Vol. 3, No. 5, October 2011

[15] Hussain I., Hussain S., Khokhar, I., Iqbal, R., "OFDMA as the Technology for the Next Generation Mobile Wireless Internet" Third International Conference on Wireless and Mobile Communications (ICWMC), Guadeloupe, 2007

[16] Roy You and J.M. Kahn, "Upper Bounding the Capacity of Optical IM/DD Channels with Multiple-Subcarrier Modulation and Fixed Bias Using Trigonometric Moment Space Method", IEEE Trans. on Information Theory, vol. 48, Issue 2, February 2002.

[17] Gamage P.A., Nirmalathas A., Lim, C., Novak D., Waterhouse, R., "Design and Analysis of Digitized RF-Over-Fiber Links" Journal of lightwave technology, Volume: 27, Issue: 12, pp. 2052-2061, 2009

[18] Wei Huang, Jiro Takayanagi, Tetsuo Sakanaka, Masao Nakagawa, "Atmospheric Optical Communication System Using Subcarrier PSK modulation", IEICE Transactions on Communications Vol.E76-B No.9 pp.1169-1177, Sep. 1993.

[19] Yoshio Karasawa, Radiowave Propagation Fundamentals for Digital Mobile Communication, Japan: Corona Publishing CO.,LTD., Tokyo, August , 2006 (in Japanese)

\section{Authors}

Jiang Liu received her B.E. degree in electronics engineering from Chong Qing University of Technology in 2001 and M.S. degree in information and telecommunications from the Graduate School of Global Information and Telecommunication Studies (GITS), Waseda University, Japan, in 2006. She is currently a Research Associate in Waseda University. Her research interests are optical communication and mobile communication.

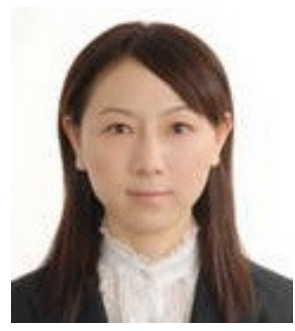

Wasinee Noonpakdee received her B.E. degree in electronics engineering from Chulalongkorn University, Thailand, in 1999 and M.ISM. degree in information systems management from Carnegie Mellon University, USA, in 2004. From 1999 to 2003, she worked as an engineer in True Corporation, a telecommunication enterprise in Thailand. From 2004 to 2008, she worked as a computer science officer, Department of Corrections, Ministry of Justice, Thailand. She is currently a Ph.D. student at the Graduate School of Global Information and Telecommunication Studies (GITS), Waseda University, Japan. Her research interests include optical wireless communications, RFID, and wireless sensor networks.

Shigeru Shimamoto received the B.E. and M.E. degrees from the University of Electro-Communications, Tokyo, Japan, in 1985 and 1987, respectively. He received the Ph.D. degree from Tohoku University, Sendai, Japan, in 1992. He joined NEC Corporation from 1987 to 1991. From 1991 to 1992, he was a research associate in the University of Electro-Communications, Tokyo, Japan. He was a research associate in Gunma University, Gunma, Japan, from 1992 to 1993. From 1994 to 2000, he was an associate professor in the Graduate School of Global Information and Telecommunication Studies (GITS), Waseda University, Tokyo, Japan. Since 2001, he has been a professor in the Graduate

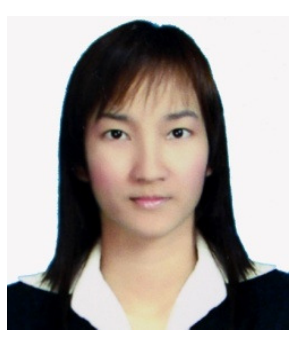
School of GITS, Waseda University. He was a visiting professor of E.E. at Stanford University in 2008. His main fields of research interests include satellite communications, mobile communications, optical wireless communications, ad-hoc networks, sensor networks, and body area networks. 